\title{
$\mu$-pseudo almost automorph mild solutions to the fractional integro-differential equation with uniform continuity
}

\section{Chuan-Yun Gu ${ }^{1,2}$ and Hong-Xu Li ${ }^{* *}$}

"Correspondence:

hoxuli@scu.edu.cn

${ }^{1}$ Department of Mathematics,

Sichuan University, Chengdu, P.R.

China

Full list of author information is

available at the end of the article

\section{Springer}

\begin{abstract}
Our aim in the article is to study the existence of $\mu$-pseudo almost automorph mild solutions to the following fractional integro-differential equation:$$
D^{\alpha} u(t)=A u(t)+\int_{-\infty}^{t} a(t-s) A u(s) d s+f(t, u(t)), \quad t \in \mathbb{R},
$$

where for $\alpha>0$, the fractional derivative $D^{\alpha}$ is understood in the sense of Weyl, and $A$ is a closed linear operator defined on Banach space $\mathbb{X}, a \in L_{\text {loc }}^{1}\left(\mathbb{R}_{+}\right)$is a scalar-valued kernel. The novelty of this work is a study of this equation with a $\mu$-SP-pseudo almost automorph nonlinear term satisfying the condition of "uniform continuity" instead of some "Lipschitz" type conditions supposed in the literature. We utilize Schauder's fixed point theorem. An example is provided to explain our abstract results.
\end{abstract}

Keywords: Mild solutions; $\mu$ - $S^{P}$-pseudo almost automorphy; Fixed point theorem; Fractional integro-differential equation

\section{Introduction}

Fractional calculus is a mathematics field for dealing with derivatives and integrals of arbitrary orders. As a result of the intensive development of fractional calculus, fractional differential equations have been proved to be useful tools in modeling of phenomena in various fields of science and economics and have been greatly developed (see [1-5] and the references therein).

In recent decades, the asymptotic properties of mild solutions for various (fractional) differential equations and (fractional) integro-differential equations have attracted much attention. Bochner first presented the notion of almost automorphy in [6] as a natural extension of almost periodicity. Since then, this notion has been promoted in a variety of ways, for example, in terms of pseudo almost automorphy ([7, 8]), weighted pseudo almost automorphy (abbr. wpaa) ([9]), $S^{p}$-weighted pseudo almost automorphy (abbr. $S^{p}$-wpaa) ([10]), $\mu$-pseudo almost automorphy (abbr. $\mu$-paa) ([11]), etc. The above-mentioned notions have been extensively applied to the research about a variety of (fractional) differential equations and (fractional) integro-differential equations (see [12-19] and the references therein)

(c) The Author(s) 2018. This article is distributed under the terms of the Creative Commons Attribution 4.0 International License (http://creativecommons.org/licenses/by/4.0/), which permits unrestricted use, distribution, and reproduction in any medium, provided you give appropriate credit to the original author(s) and the source, provide a link to the Creative Commons license, and indicate if changes were made. 
In particular, Ponce [18] studied the existence and uniqueness of bounded solutions, such as almost periodic (automorphic) and asymptotically almost periodic solution, etc., to the following fractional integro-differential equation:

$$
D^{\alpha} v(t)=A v(t)+\int_{-\infty}^{t} a(t-s) A v(s) d s+f(t, v(t)), \quad t \in \mathbb{R},
$$

where $D^{\alpha}$ is comprehended as a fractional derivative of order $\alpha>0$ in the sense of Weyl (see $[4,18])$ and $A$ is a linear and closed operator defined in a Banach space $\mathbb{X}, a \in L_{\text {loc }}^{1}\left(\mathbb{R}_{+}\right)$ is a scalar-valued kernel, and $f: \mathbb{R} \times \mathbb{X} \rightarrow \mathbb{X}$ belongs to a closed subspace of the space of continuous and bounded functions satisfying some "Lipschitz" type conditions. Subsequently, Chang [19] investigated some existence results about wpaa solutions to Eq. (1.1) where the nonlinear term $f$ is a $S^{p}$-wpaa function satisfying a number of conditions of "Lipschitz" type combined with the contraction map theorem or a "uniform continuity" type condition combined with the Leray-Schauder alternative theorem. From the literature mentioned above and to the best of our knowledge, there is no work about asymptotic properties of mild solutions to Eq. (1.1) where the nonlinear term $f$ satisfies a "uniform continuity" type condition combined with Schauder's fixed point theorem. This is a motivation of writing this manuscript.

Recently, by using the measure theory, Chang [20] and Abdelkarim-Nidal Akdad [21] presented the notion of $\mu-S^{p}$-pseudo almost automorphy (abbr. $\mu-S^{p}$-paa), which is a generalization of a $\mu$-pseudo almost automorphic function, respectively. The natural question is raised: what are asymptotic properties of mild solutions about Eq. (1.1) where the nonlinear term $f$ is a $\mu$-S $S^{p}$-paa function? To the best of our knowledge, there is rarely literature covering the existence of $\mu$-paa solutions about Eq. (1.1) where the nonlinear term $f$ is a $\mu$ $S^{p}$-paa function. To close this gap, by utilizing Schauder's fixed point theorem, we obtain $\mu$-paa mild solutions for Eq. (1.1) with the $\mu-S^{p}$-paa nonlinear term $f$ satisfying the condition of "uniform continuity" type instead of some "Lipschitz" type conditions supposed in the literature.

The rest of this article is organized as follows. In Sect. 2, we recall some basic definitions and lemmas, which are based on the literature. In Sect. 3, we present our main results, namely, the existence of $\mu$-paa mild solutions to Eq. (1.1). These results are based on the nonlinear term $f$ that satisfies a "uniform continuity" type condition combined with Schauder's fixed point theorem. The last section is dedicated to the application of our results. An example is provided to explain our abstract results, where the condition of "uniform continuity" type is satisfied but the condition of "Lipschitz" type failed.

\section{Preliminaries}

Let us review the notation. $(\mathbb{X},\|\cdot\|)$ and $(\mathbb{Y},\|\cdot\|)$ are Banach spaces. The space $\mathrm{BC}(\mathbb{R}, \mathbb{X})=$ $\{v: \mathbb{R} \rightarrow \mathbb{X}: v$ is a bounded and continuous function $\}$ is a Banach space with the supremum norm.

Throughout this article, the Lebesgue $\sigma$-field of $\mathbb{R}$ is denoted by $\mathcal{C}$ and the set consisting of whole positive measures $\mu$ on $\mathcal{C}$ such that $\mu(\mathbb{R})=+\infty$ and $\mu([c, d])<+\infty$ for any $c, d \in \mathbb{R}$ $(c<d)$ is denoted by $\mathfrak{W}$. In the article, we always suppose that $\mu \in \mathfrak{W}$. 


\section{Definition 2.1 ([11])}

(i) A continuous and bounded function $f: \mathbb{R} \rightarrow \mathbb{X}$ is called $\mu$-ergodic if

$$
\lim _{S \rightarrow+\infty} \frac{1}{\mu([-S, S])} \int_{[-S, S]}\|f(t)\| d \mu=0
$$

The space formed by all these functions is denoted by $\varepsilon(\mathbb{X}, \mu)$. The space PAA $(\mathbb{X}, \mu)$ formed by all $\mu$-paa functions is given by

$$
\operatorname{PAA}(\mathbb{X}, \mu)=\left\{f=f_{1}+f_{2} \in \mathrm{BC}(\mathbb{R}, \mathbb{X}): f_{1} \in \mathrm{AA}(\mathbb{X}), f_{2} \in \varepsilon(\mathbb{X}, \mu)\right\}
$$

(ii) A continuous and bounded function $f: \mathbb{R} \times \mathbb{Y} \rightarrow \mathbb{X}$ is called $\mu$-ergodic if $f(\cdot, v)$ is bounded for any $v \in \mathbb{Y}$ and

$$
\lim _{S \rightarrow+\infty} \frac{1}{\mu([-S, S])} \int_{[-S, S]}\|f(t, v)\| d \mu=0
$$

uniformly in $v \in \mathbb{Y}$. The space formed by all these functions is denoted by $\varepsilon(\mathbb{R} \times \mathbb{Y}, \mathbb{X}, \mu)$.

The space $\operatorname{PAA}(\mathbb{R} \times \mathbb{Y}, \mathbb{X}, \mu)$ formed by all $\mu$-paa functions is given by

$$
\begin{aligned}
& \operatorname{PAA}(\mathbb{R} \times \mathbb{Y}, \mathbb{X}, \mu) \\
& \quad=\left\{f=f_{1}+f_{2} \in \mathrm{BC}(\mathbb{R} \times \mathbb{Y}, \mathbb{X}): f_{1} \in \mathrm{AA}(\mathbb{R} \times \mathbb{Y}, \mathbb{X}), f_{2} \in \varepsilon(\mathbb{R} \times \mathbb{Y}, \mathbb{X}, \mu)\right\}
\end{aligned}
$$

Definition $2.2([14])$ The space $\mathrm{BS}^{p}(\mathbb{X})$ formed by the whole Stepanov bounded functions, where $p \in[1, \infty)$, includes of the whole measurable functions $f: \mathbb{R} \rightarrow \mathbb{X}$ satisfying $f^{b} \in L^{\infty}\left(\mathbb{R}, L^{p}(0,1 ; \mathbb{X})\right)$. It is a Banach space where its norm is defined by

$$
\|f\|_{S^{p}}=\left\|f^{b}\right\|_{L^{\infty}\left(\mathbb{R}, L^{p}\right)}=\sup _{t \in \mathbb{R}}\left(\int_{t}^{t+1}\|f(\tau)\|^{p} d \tau\right)^{1 / p}=\sup _{t \in \mathbb{R}}\|f(t+\cdot)\|_{p} .
$$

\section{Definition 2.3 ([14])}

(i) The space $\mathrm{AS}^{p}(\mathbb{X})$ formed by whole $S^{p}$-aa functions, includes of all $f \in \operatorname{BS}^{p}(\mathbb{X})$ satisfying $f^{b} \in \mathrm{AA}\left(L^{p}(0,1 ; \mathbb{X})\right)$.

(ii) A function $f \in \mathrm{BS}^{p}(\mathbb{R} \times \mathbb{Y}, \mathbb{X})$ is called $S^{p}$-aa in $t \in \mathbb{R}$ for $v \in \mathbb{Y}$, if $f(\cdot, v) \in \mathrm{AS}^{p}(\mathbb{X})$ for $v \in \mathbb{Y}$. The set consisting of the whole of these functions is denoted by $\operatorname{AS}^{p}(\mathbb{R} \times \mathbb{Y}, \mathbb{X})$.

From $[20,21]$, the spaces $\operatorname{PAA}^{p}(\mathbb{X}, \mu)$ and $\operatorname{PAA}^{p}(\mathbb{R} \times \mathbb{Y}, \mathbb{X}, \mu)$ consisting of the whole $\mu-S^{p}$-paa functions are defined by

$$
\operatorname{PAA}^{p}(\mathbb{X}, \mu)=\left\{f=f_{1}+f_{2} \in \mathrm{BS}^{p}(\mathbb{X}): f_{1} \in \mathrm{AS}^{p}(\mathbb{X}), f_{2}^{b} \in \varepsilon\left(L^{p}(0,1 ; \mathbb{X}), \mu\right)\right\}
$$

where $\varepsilon\left(L^{p}(0,1 ; \mathbb{X}), \mu\right)$, which is $f_{2}^{b} \in \mathrm{BC}\left(L^{p}(0,1 ; \mathbb{X})\right)$ and

$$
\lim _{S \rightarrow+\infty} \frac{1}{\mu([-S, S])} \int_{[-S, S]}\left(\int_{t}^{t+1}\left\|f_{2}(s)\right\|^{p} d s\right)^{\frac{1}{p}} d \mu=0 .
$$


and

$$
\begin{aligned}
& \operatorname{PAA}^{p}(\mathbb{R} \times \mathbb{Y}, \mathbb{X}, \mu)=\left\{f=f_{1}+f_{2}: f_{1} \in \mathrm{AS}^{p}(\mathbb{R} \times \mathbb{Y}, \mathbb{X}),\right. \\
&\left.f_{2}^{b} \in \varepsilon\left(\mathbb{Y}, L^{p}(0,1 ; \mathbb{X}), \mu\right), f(\cdot, v) \in L_{\text {loc }}^{p}(\mathbb{R}, \mathbb{X}) \text { for each } v \in \mathbb{Y}\right\} .
\end{aligned}
$$

Let the positive measure on $\mathcal{C}$, denoted by $\mu_{\varsigma}$, be defined as

$$
\mu_{\varsigma}(A)=\mu(\{a+\varsigma: a \in A\}) \quad \text { for } A \in \mathcal{C}, \varsigma \in \mathbb{R} .
$$

The following assumption [11] will be needed later.

(A) For $\forall \varsigma \in \mathbb{R}$, there are a bounded interval $\Omega$ and a constant $\gamma>0$ satisfying

$$
\mu_{\varsigma}(A) \leq \gamma \mu(A)
$$

when $A \in \mathcal{C}$ satisfies $A \cap \Omega=\emptyset$.

Lemma $2.1([11,20,21])$ If the assumption $(\mathrm{A})$ holds, then $\varepsilon(\mathbb{X}, \mu)$ and $\varepsilon\left(L^{p}(0,1 ; \mathbb{X}), \mu\right)$ are translation invariant.

Consequently, $\operatorname{PAA}(\mathbb{X}, \mu)$ and $\operatorname{PAA}^{p}(\mathbb{X}, \mu)$ are also translation invariant.

Lemma $2.2([20,21])$ If the assumption (A) holds, then $\mathrm{PAA}(\mathbb{X}, \mu) \subset \mathrm{PAA}^{p}(\mathbb{X}, \mu)$ for each $1 \leq p<\infty$.

\section{Main results}

Now, we address the existence of $\mu$-paa mild solutions to Eq. (1.1). Our existence theorem is based upon the nonlinear term $f \in \operatorname{PAA}^{p}(\mathbb{R} \times \mathbb{X}, \mathbb{X}, \mu)$ satisfying a "uniform continuity" type condition in the place of some "Lipschitz" type conditions supposed in the literature combined with Schauder's fixed point theorem.

We recall that the space formed by whole linear and bounded operators from $\mathbb{X}$ to $\mathbb{Y}$ is denoted by $\mathfrak{B}(\mathbb{X}, \mathbb{Y}), \mathfrak{B}(\mathbb{X}):=\mathfrak{B}(\mathbb{X}, \mathbb{X})$ for short.

Definition 3.1 ([18]) If $A$ is a closed and linear operator where domain $D(A)$ is defined in Banach space $\mathbb{X}, \alpha>0$ and $a \in L_{\text {loc }}^{1}\left(\mathbb{R}_{+}\right)$, there are a strongly continuous functions $S_{\alpha}$ : $[0, \infty) \rightarrow \mathfrak{B}(\mathbb{X})$ and $\omega \geq 0$ satisfying $\left\{\frac{\lambda^{\alpha}}{1+\hat{a}(\lambda)}: \operatorname{Re} \lambda>\omega\right\} \subset \bar{\rho}(A)$ and, for any $x \in \mathbb{X}$,

$$
\left(\lambda^{\alpha}-(1+\hat{a}(\lambda)) A\right)^{-1} x=\frac{1}{1+\hat{a}(\lambda)}\left(\frac{\lambda^{\alpha}}{1+\hat{a}(\lambda)}-A\right)^{-1} x=\int_{0}^{\infty} e^{-\lambda t} S_{\alpha}(t) x d t, \quad \operatorname{Re} \lambda>\omega,
$$

then the operator $A$ is said to be the generator of an $\alpha$-resolvent family, where the resolvent set of $A$ and the Laplace transform of $a$ are denoted by $\bar{\rho}(A)$ and $\hat{a}$, respectively. In such a situation, $\left\{S_{\alpha}(t)\right\}_{t \geq 0}$ is said to be the $\alpha$-resolvent family generated by $A$.

Lemma 3.1 ([22]) Iffor all $t>0, S_{\alpha}(t)$ is a continuous and compact operator in the uniform operator topology, then $\lim _{h \rightarrow 0}\left\|S_{\alpha}(t+h)-S_{\alpha}(h) S_{\alpha}(t)\right\|=0$ and $\lim _{h \rightarrow 0} \| S_{\alpha}(t)-S_{\alpha}(h) S_{\alpha}(t-$ h) $\|=0$ for all $t>0$. 
Definition 3.2 ([18]) Let $\alpha>0$ and $A$ be the generator of an $\alpha$-resolvent family $\left\{S_{\alpha}(t)\right\}_{t \geq 0}$. For a function $v \in C(\mathbb{R}, \mathbb{X})$, if the function $s \mapsto S_{\alpha}(t-s) f(s, v(s))$ is integrable on $(-\infty, t)$ for each $t \in \mathbb{R}$ and

$$
v(t)=\int_{-\infty}^{t} S_{\alpha}(t-s) f(s, v(s)) d s
$$

then the function $v$ is called a mild solution of Eq. (1.1).

We will use the following assumptions:

(A $\left.\mathrm{A}_{1}\right) A$ generates an $\alpha$-resolvent family $\left\{S_{\alpha}(t)\right\}_{t \geq 0}$ satisfying $\left\|S_{\alpha}(t)\right\| \leq \varphi_{\alpha}(t), \forall t \geq 0$, where $\varphi_{\alpha}(t) \in L^{1}\left(\mathbb{R}_{+}\right)$is nonincreasing in $t$ satisfying $\varphi_{0}:=\sum_{n=0}^{\infty} \varphi_{\alpha}(n)<\infty$.

$\left(\mathrm{A}_{2}\right)$ The function $f=f_{1}+f_{2} \in \mathrm{PAA}^{p}(\mathbb{R} \times \mathbb{X}, \mathbb{X}, \mu)$ where for each bounded subset $\mathbb{B} \subset \mathbb{X}, f_{1} \in \mathrm{AS}^{p}(\mathbb{R} \times \mathbb{X}, \mathbb{X})$ is uniform continuity uniformly in $t \in \mathbb{R}$ and $f_{2}^{b} \in$ $\varepsilon\left(\mathbb{X}, L^{p}(0,1 ; \mathbb{X}), \mu\right)$.

$\left(\mathrm{A}_{3}\right) f \in \operatorname{PAA}^{p}(\mathbb{R} \times \mathbb{X}, \mathbb{X}, \mu)$ and, for each bounded subset $\mathbb{B} \subset \mathbb{X}, f(t, v)$ is uniform continuity uniformly in $t \in \mathbb{R}$ and $\{f(\cdot, v): v \in \mathbb{B}\}$ is bounded in $\operatorname{PAA}^{p}(\mathbb{R} \times \mathbb{X}, \mathbb{X}, \mu)$ for each bounded subset $\mathbb{B} \subset \mathbb{X}$.

For $v \in \operatorname{PAA}(\mathbb{X}, \mu)$, record

$$
U v=\int_{-\infty}^{t} S_{\alpha}(t-s) f(s, v(s)) d s=\int_{0}^{\infty} S_{\alpha}(s) f(t-s, v(t-s)) d s
$$

Lemma 3.2 If $(\mathrm{A})$ and $\left(\mathrm{A}_{1}\right)-\left(\mathrm{A}_{3}\right)$ hold, then $U: \operatorname{PAA}(\mathbb{X}, \mu) \rightarrow \operatorname{PAA}(\mathbb{X}, \mu)$ is continuous.

Proof For $\chi \in \mathrm{BS}^{p}(\mathbb{X})$ and $t \in \mathbb{R}$, by $\left(\mathrm{A}_{1}\right)$, we have

$$
\begin{aligned}
\left\|\int_{0}^{\infty} S_{\alpha}(\varsigma) \chi(t-\varsigma) d \varsigma\right\| & \leq \sum_{k=0}^{\infty} \int_{k}^{k+1}\left\|S_{\alpha}(\varsigma) \chi(t-\varsigma)\right\| d \varsigma \\
& \leq \sum_{k=0}^{\infty} \int_{k}^{k+1}\left\|S_{\alpha}(\varsigma)\right\|\|\chi(t-\varsigma)\| d \varsigma \\
& \leq \sum_{k=0}^{\infty} \int_{k}^{k+1} \varphi_{\alpha}(\varsigma)\|\chi(t-\varsigma)\| d \varsigma \\
& \leq \sum_{k=0}^{\infty} \varphi_{\alpha}(k) \int_{k}^{k+1}\|\chi(t-\varsigma)\| d \varsigma \\
& \leq \sum_{k=0}^{\infty} \varphi_{\alpha}(k)\left(\int_{k}^{k+1}\|\chi(t-\varsigma)\|^{p} d \varsigma\right)^{\frac{1}{p}} \\
& =\sum_{k=0}^{\infty} \varphi_{\alpha}(k)\|\chi(t+k-1+\cdot)\|_{p} \\
& \leq \varphi_{0}\|\chi\|_{S^{p}} .
\end{aligned}
$$

If $v \in \operatorname{PAA}(\mathbb{X}, \mu)$, then $f(t, v(t)) \in \operatorname{PAA}^{p}(\mathbb{X}, \mu)$ by Theorem 3.3 of [20] and Lemma 2.2 and we record $\psi(t)=f(t, v(t)), t \in \mathbb{R}$. Let $\psi=\psi_{1}+\psi_{2}$ with $\psi_{1} \in \mathrm{AS}^{p}(\mathbb{X})$ and $\psi_{2}^{b} \in$ 
$\varepsilon\left(L^{p}(0,1 ; \mathbb{X}), \mu\right)$. For $t \in \mathbb{R}, i=1,2$, we denote

$$
\Psi_{i}(t)=\int_{0}^{\infty} S_{\alpha}(\varsigma) \psi_{i}(t-\varsigma) d \varsigma
$$

By (3.1), for $t, s \in \mathbb{R}$, we have

$$
\begin{aligned}
& \left\|\Psi_{i}(t)\right\| \leq \varphi_{0}\left\|\psi_{i}\right\|_{S^{p}} \\
& \left\|\Psi_{i}(t)-\Psi_{i}(s)\right\| \leq \sum_{k=0}^{\infty} \varphi_{\alpha}(k)\left\|\psi_{i}(t+k-1+\cdot)-\psi_{i}(s+k-1+\cdot)\right\|_{p}
\end{aligned}
$$

Notice that, for $t, s \in \mathbb{R}, \sum_{k=0}^{\infty} \varphi_{\alpha}(k)\left\|\psi_{i}(t+k-1+\cdot)-\psi_{i}(s+k-1+\cdot)\right\|_{p}$ is uniformly convergent. So $\Psi_{i} \in \mathrm{BC}(\mathbb{R}, \mathbb{X})$. At present, the proof is achieved in the following three steps.

Step 1. Since $\psi_{1} \in \operatorname{AS}^{p}(\mathbb{X})$, for $\left\{s_{n}^{\prime}\right\} \subset \mathbb{R}$ and $t \in \mathbb{R}$, there is $\left\{s_{n}\right\} \subset\left\{s_{n}^{\prime}\right\}$ and a function $\hat{\psi}_{1} \in L_{\mathrm{loc}}^{p}(\mathbb{R}, \mathbb{X})$ satisfying

$$
\lim _{n \rightarrow \infty}\left\|\psi_{1}\left(t+s_{n}+\cdot\right)-\hat{\psi}_{1}(t+\cdot)\right\|_{p}=\lim _{n \rightarrow \infty}\left\|\hat{\psi}_{1}\left(t-s_{n}+\cdot\right)-\psi_{1}(t+\cdot)\right\|_{p}=0
$$

Let

$$
\hat{\Psi}_{1}(t)=\int_{0}^{\infty} S_{\alpha}(\varsigma) \hat{\psi}_{1}(t-\varsigma) d \varsigma, \quad t \in \mathbb{R}
$$

It is easy to see that $\sum_{k=0}^{\infty} \varphi_{\alpha}(k)\left\|\psi_{1}\left(t+s_{n}+k-1+\cdot\right)-\hat{\psi}_{1}(t+k-1+\cdot)\right\|_{p}$ is uniformly convergent in $t \in \mathbb{R}$. For $t \in \mathbb{R}$, by (3.1) and (3.2), we have

$$
\begin{aligned}
& \left\|\Psi_{1}\left(t+s_{n}\right)-\hat{\Psi}_{1}(t)\right\| \\
& \quad=\left\|\int_{0}^{\infty} S_{\alpha}(\varsigma)\left(\psi_{1}\left(t+s_{n}-\varsigma\right)-\psi_{1}(t-\varsigma)\right) d \varsigma\right\| \\
& \quad \leq \sum_{k=0}^{\infty} \varphi_{\alpha}(k)\left\|\psi_{1}\left(t+s_{n}+k-1+\cdot\right)-\hat{\psi}_{1}(t+k-1+\cdot)\right\|_{p} \\
& \quad \rightarrow 0 \text { as } n \rightarrow \infty .
\end{aligned}
$$

Analogously, we are also able to testify that

$$
\lim _{n \rightarrow \infty}\left\|\hat{\Psi}_{1}\left(t-s_{n}\right)-\Psi_{1}(t)\right\|=0 \quad \text { for } t \in \mathbb{R}
$$

This implies that $\Psi_{1} \in \mathrm{AA}(\mathbb{X})$.

Step 2. Since (A) holds, we obtain

$$
\begin{aligned}
& \lim _{S \rightarrow \infty} \frac{1}{\mu([-S, S])} \int_{[-S, S]}\left(\int_{k}^{k+1}\left\|\psi_{2}(t-\varsigma)\right\|^{p} d \varsigma\right)^{\frac{1}{p}} d \mu \\
& \quad=\lim _{S \rightarrow \infty} \frac{1}{\mu([-S, S])} \int_{[-S, S]}\left\|\psi_{2}(t+k-1+\cdot)\right\|_{p} d \mu=0, \quad k=1,2, \ldots
\end{aligned}
$$


Obviously, $\sum_{k=0}^{\infty} \varphi_{\alpha}(k)\left\|\psi_{2}(t+k-1+\cdot)\right\|_{p}$ is uniformly convergent in $t \in \mathbb{R}$ and

$$
\sum_{k=0}^{\infty} \varphi_{\alpha}(k) \frac{1}{\mu([-S, S])} \int_{[-S, S]}\left(\int_{k}^{k+1}\left\|\psi_{2}(t-\varsigma)\right\|^{p} d \varsigma\right)^{\frac{1}{p}} d \mu
$$

is uniformly convergent in $S \in(0, \infty)$. By (3.1),

$$
\begin{aligned}
& \frac{1}{\mu([-S, S])} \int_{[-S, S]}\left\|\Psi_{2}(t)\right\| d \mu \\
& \quad=\frac{1}{\mu([-S, S])} \int_{[-S, S]}\left\|\int_{0}^{\infty} S_{\alpha}(\varsigma) \psi_{2}(t-\varsigma) d \varsigma\right\| d \mu \\
& \quad \leq \frac{1}{\mu([-S, S])} \int_{[-S, S]}\left[\sum_{k=0}^{\infty} \varphi_{\alpha}(k)\left\|\psi_{2}(t+k-1+\cdot)\right\|_{p}\right] d \mu \\
& =\sum_{k=0}^{\infty} \varphi_{\alpha}(k) \frac{1}{\mu([-S, S])} \int_{[-S, S]}\left(\int_{k}^{k+1}\left\|\psi_{2}(t-\varsigma)\right\|^{p} d \varsigma\right)^{\frac{1}{p}} d \mu \\
& \quad \rightarrow 0 \quad \text { as } S \rightarrow \infty .
\end{aligned}
$$

This implies that $\Psi_{2} \in \varepsilon(\mathbb{X}, \mu)$.

Step 3. For $\epsilon>0$ and $u, v \in \operatorname{PAA}(\mathbb{X}, \mu)$, there is $\sigma>0$ such that $\|u-v\|<\sigma$. By $\left(\mathrm{A}_{3}\right)$, we obtain $\|f(t, u(t))-f(t, v(t))\|<\epsilon$ for $t \in \mathbb{R}$ and record $\kappa(t)=f(t, u(t))-f(t, v(t)), t \in \mathbb{R}$, thus $\|\kappa\|_{S^{p}} \leq \epsilon$. Thus from (3.1), we have

$$
\|U u-U v\|=\sup _{t \in \mathbb{R}}\left\|\int_{0}^{\infty} S_{\alpha}(\varsigma) \kappa(t-\varsigma) d \varsigma\right\| \leq \varphi_{0}\|\kappa\|_{S^{p}} \leq \varphi_{0} \epsilon .
$$

This implies that $U: \operatorname{PAA}(\mathbb{X}, \mu) \rightarrow \operatorname{PAA}(\mathbb{X}, \mu)$ is uniformly continuous.

We provide some hypotheses which will be applied below:

$\left(\mathrm{A}_{4}\right)$ There is $r>0$ satisfying $\|f(t, v)\|_{S^{p}} \leq \frac{r}{\varphi_{0}}$ for $v \in \operatorname{PAA}(\mathbb{X}, \mu)$ with $\|v\| \leq r$.

$\left(\mathrm{A}_{5}\right)$ Let $\left\{v_{n}\right\}$ be a bounded sequence in $\mathrm{PAA}(\mathbb{X}, \mu)$ and uniform continuity in any compact subset of $\mathbb{R}$. Then $\left\{f\left(\cdot, v_{n}(\cdot)\right)\right\}$ is relatively compact in $\operatorname{PAA}^{p}(\mathbb{X}, \mu)$.

Theorem 3.1 If $S_{\alpha}(t)$ is a continuous and compact operator for all $t>0$ in the uniform operator topology, then under assumptions $(\mathrm{A})$ and $\left(\mathrm{A}_{1}\right)-\left(\mathrm{A}_{5}\right)$, Eq. (1.1) has a $\mu$-paa mild solution.

Proof Let $B_{r}:=\{v \in \operatorname{PAA}(\mathbb{X}, \mu):\|v\| \leq r\}$. Then $B_{r}$ is a convex and closed subset of $\operatorname{PAA}(\mathbb{X}, \mu)$. The proof can be carried out via a four-step process.

Step 1: For $r>0$, we can obtain $\mathrm{UB}_{r} \subset B_{r}$. For $v \in B_{r}, t \in \mathbb{R}$, by $\left(\mathrm{A}_{1}\right)$ and $\left(\mathrm{A}_{4}\right)$, then

$$
\begin{aligned}
\|U \nu(t)\| & =\left\|\int_{-\infty}^{t} S_{\alpha}(t-s) f(s, v(s)) d s\right\| \\
& \leq \sum_{n=1}^{\infty}\left\|\int_{t-n}^{t-n+1} S_{\alpha}(t-s) f(s, v(s)) d s\right\| \\
& \leq \sum_{n=1}^{\infty} \int_{t-n}^{t-n+1}\left\|S_{\alpha}(t-s)\right\|\|f(s, v(s))\| d s
\end{aligned}
$$




$$
\begin{aligned}
& \leq \sum_{n=1}^{\infty} \int_{t-n}^{t-n+1} \varphi_{\alpha}(t-s)\|f(s, v(s))\| d s \\
& \leq \sum_{n=1}^{\infty} \varphi_{\alpha}(n-1)\left(\int_{t-n}^{t-n+1}\|f(s, v(s))\|^{p} d s\right)^{\frac{1}{p}} \\
& \leq \sum_{n=0}^{\infty} \varphi_{\alpha}(n)\|f(\cdot, v(\cdot))\|_{S^{p}} \\
& =\varphi_{0}\|f(\cdot, v(\cdot))\|_{S^{p}} \\
& \leq r .
\end{aligned}
$$

Thus $\mathrm{UB}_{r} \subset B_{r}$.

Step 2: For $v \in B_{r}$, by $\left(\mathrm{A}_{4}\right)$ and (3.1), we have

$$
\|U v\|=\sup _{t \in \mathbb{R}}\left\|\int_{0}^{\infty} S_{\alpha}(\varsigma) f(t-\varsigma, v(t-\varsigma)) d \varsigma\right\| \leq \varphi_{0}\|f(\cdot, v(\cdot))\|_{S^{p}} \leq r .
$$

Then $U: B_{r} \rightarrow B_{r}$ is continuous by Lemma 3.2.

Step 3: $\left\{U v: v \in B_{r}\right\} \subset \operatorname{PAA}(\mathbb{X}, \mu)$ is equi-continuous. Let $q>1$ satisfy $\frac{1}{p}+\frac{1}{q}=1$ and take $t_{1}, t_{2} \in \mathbb{R}$ with $t_{1}>t_{2}$ and $0<\epsilon<1$ such that $\eta=\min \left\{1-\left(\frac{\epsilon}{12 r}\right)^{q},\left(\frac{\epsilon}{12 r}\right)^{q}\right\} \leq 1$. For $v \in B_{r}$, with $r>0$ and $t_{1}-t_{2}<\eta$, we can decompose $U \nu\left(t_{1}\right)-U v\left(t_{2}\right)=I_{1}+I_{2}+I_{3}$, where

$$
\begin{aligned}
& I_{1}=\int_{t_{2}}^{t_{1}} S_{\alpha}\left(t_{1}-s\right) f(s, v(s)) d s, \\
& I_{2}=\int_{t_{2}-\eta}^{t_{2}}\left[S_{\alpha}\left(t_{1}-s\right)-S_{\alpha}\left(t_{2}-s\right)\right] f(s, v(s)) d s, \\
& I_{3}=\int_{-\infty}^{t_{2}-\eta}\left[S_{\alpha}\left(t_{1}-s\right)-S_{\alpha}\left(t_{2}-s\right)\right] f(s, v(s)) d s .
\end{aligned}
$$

By $\left(A_{1}\right)$ and $\left(A_{4}\right)$, we have

$$
\begin{aligned}
\left\|I_{1}\right\| & \leq \int_{t_{2}}^{t_{1}}\left\|S_{\alpha}\left(t_{1}-s\right)\right\|\|f(s, v(s))\| d s \\
& \leq \int_{t_{2}}^{t_{1}} \varphi_{\alpha}\left(t_{1}-s\right)\|f(s, v(s))\| d s \\
& \leq\left(\int_{t_{2}}^{t_{1}} \varphi_{\alpha}^{q}\left(t_{1}-s\right) d s\right)^{\frac{1}{q}} \cdot\left(\int_{t_{2}}^{t_{1}}\|f(s, v(s))\|^{p} d s\right)^{\frac{1}{p}} \\
& \leq \varphi_{0} \cdot \eta^{\frac{1}{q}}\|f(\cdot, v(\cdot))\|_{S^{p}} \\
& \leq \varphi_{0} \cdot \eta^{\frac{1}{q}} \cdot \frac{r}{\varphi_{0}} \\
& \leq \frac{\epsilon}{6}
\end{aligned}
$$




$$
\begin{aligned}
\left\|I_{2}\right\| & \leq \int_{t_{2}-\eta}^{t_{2}}\left\|S_{\alpha}\left(t_{1}-s\right)-S_{\alpha}\left(t_{2}-s\right)\right\|\|f(s, v(s))\| d s \\
& \leq \int_{t_{2}-\eta}^{t_{2}}\left(\varphi_{\alpha}\left(t_{1}-s\right)+\varphi_{\alpha}\left(t_{2}-s\right)\right)\|f(s, v(s))\| d s \\
& \leq\left(\int_{t_{2}-\eta}^{t_{2}}\left(\varphi_{\alpha}\left(t_{1}-s\right)+\varphi_{\alpha}\left(t_{2}-s\right)\right)^{q} d s\right)^{\frac{1}{q}} \cdot\left(\int_{t_{2}-\eta}^{t_{2}}\|f(s, v(s))\|^{p} d s\right)^{\frac{1}{p}} \\
& \leq 2 \varphi_{0} \cdot \eta^{\frac{1}{q}}\|f(\cdot, v(\cdot))\|_{S^{p}} \\
& \leq 2 \varphi_{0} \cdot \eta^{\frac{1}{q}} \cdot \frac{r}{\varphi_{0}} \\
& \leq \frac{\epsilon}{3}
\end{aligned}
$$

and

$$
\begin{aligned}
\left\|I_{3}\right\| \leq & \int_{-\infty}^{t_{2}-\eta}\left\|\left[S_{\alpha}\left(t_{1}-s\right)-S_{\alpha}\left(t_{2}-s\right)\right]\right\|\|f(s, v(s))\| d s \\
\leq & \sum_{n=1}^{\infty} \int_{t_{2}-\eta-n}^{t_{2}-\eta-n+1}\left(\varphi_{\alpha}\left(t_{1}-s\right)+\varphi_{\alpha}\left(t_{2}-s\right)\right)\|f(s, v(s))\| d s \\
\leq & \sum_{n=1}^{\infty} \int_{t_{2}-\eta-n}^{t_{2}-n}\left(\varphi_{\alpha}\left(t_{1}-s\right)+\varphi_{\alpha}\left(t_{2}-s\right)\right)\|f(s, v(s))\| d s \\
& +\sum_{n=1}^{\infty} \int_{t_{2}-n}^{t_{2}-\eta-n+1}\left(\varphi_{\alpha}\left(t_{1}-s\right)+\varphi_{\alpha}\left(t_{2}-s\right)\right)\|f(s, v(s))\| d s \\
\leq & \sum_{n=1}^{\infty}\left(\varphi_{\alpha}\left(t_{1}-t_{2}+n-1\right)+\varphi_{\alpha}(n-1)\right) \cdot \eta^{\frac{1}{q}} \cdot\|f(\cdot, v(\cdot))\|_{S^{p}} \\
& +\sum_{n=1}^{\infty}\left(\varphi_{\alpha}\left(t_{1}-t_{2}+n-1\right)+\varphi_{\alpha}(n-1)\right) \cdot(1-\eta)^{\frac{1}{q}} \cdot\|f(\cdot, v(\cdot))\|_{S^{p}} \\
\leq & 2 \varphi_{0} \cdot \eta^{\frac{1}{q}} \cdot \frac{r}{\varphi_{0}}+2 \varphi_{0} \cdot(1-\eta)^{\frac{1}{q}} \cdot \frac{r}{\varphi_{0}} \\
\leq & \frac{\epsilon}{3} .
\end{aligned}
$$

From (3.3), (3.4) and (3.5), we have

$$
\left\|U \nu\left(t_{1}\right)-U \nu\left(t_{2}\right)\right\|<\epsilon .
$$

Step 4: $\left\{(U v)(t): v \in B_{r}\right\}$ is relatively compact sets in $X$ for any $t \in \mathbb{R}$. Let there is $\epsilon \in(0,1)$, then $\left\{S_{\alpha}(\epsilon) \int_{-\infty}^{t-\epsilon} S_{\alpha}(t-s-\epsilon) f(s, v(s)) d s: v \in B_{r}\right\}$ is relatively compact since $S_{\alpha}(\epsilon)$ is compact. Furthermore, for arbitrary $\epsilon<\delta<1$, we have

$$
\begin{gathered}
\left\|S_{\alpha}(\epsilon) \int_{-\infty}^{t-\epsilon} S_{\alpha}(t-s-\epsilon) f(s, v(s)) d s-\int_{-\infty}^{t-\epsilon} S_{\alpha}(t-s) f(s, v(s)) d s\right\| \\
\quad \leq \int_{-\infty}^{t-\epsilon}\left\|S_{\alpha}(\epsilon) S_{\alpha}(t-s-\epsilon)-S_{\alpha}(t-s)\right\|\|f(s, v(s))\| d s \\
\leq \int_{-\infty}^{t-\delta}\left\|S_{\alpha}(\epsilon) S_{\alpha}(t-s-\epsilon)-S_{\alpha}(t-s)\right\|\|f(s, v(s))\| d s
\end{gathered}
$$




$$
\begin{aligned}
& +\int_{t-\delta}^{t-\epsilon}\left\|S_{\alpha}(\epsilon) S_{\alpha}(t-s-\epsilon)-S_{\alpha}(t-s)\right\|\|f(s, v(s))\| d s \\
& \leq \sum_{n=1}^{\infty} \int_{t-\delta-n}^{t-\delta-n+1}\left\|S_{\alpha}(\epsilon) S_{\alpha}(t-s-\epsilon)-S_{\alpha}(t-s)\right\|\|f(s, v(s))\| d s \\
& +\int_{t-\delta}^{t-\epsilon}\left\|S_{\alpha}(\epsilon) S_{\alpha}(t-s-\epsilon)-S_{\alpha}(t-s)\right\|\|f(s, v(s))\| d s \\
& \leq \sum_{n=1}^{\infty} \int_{t-\delta-n}^{t-\delta-n+1}\left(\varphi_{\alpha}(\epsilon) \varphi_{\alpha}(t-s-\epsilon)+\varphi_{\alpha}(t-s)\right)\|f(s, v(s))\| d s \\
& +\int_{t-\delta}^{t-\epsilon}\left(\varphi_{\alpha}(\epsilon) \varphi_{\alpha}(t-s-\epsilon)+\varphi_{\alpha}(t-s)\right)\|f(s, v(s))\| d s \\
& \leq \sum_{n=1}^{\infty}\left(\int_{t-\delta-n}^{t-\delta-n+1}\left(\varphi_{\alpha}(\epsilon) \varphi_{\alpha}(t-s-\epsilon)+\varphi_{\alpha}(t-s)\right)^{q} d s\right)^{\frac{1}{q}} \\
& \cdot\left(\int_{t-\delta-n}^{t-\delta-n+1}\|f(s, v(s))\|^{p} d s\right)^{\frac{1}{p}} \\
& +\left(\int_{t-\delta}^{t-\epsilon}\left(\varphi_{\alpha}(\epsilon) \varphi_{\alpha}(t-s-\epsilon)+\varphi_{\alpha}(t-s)\right)^{q} d s\right)^{\frac{1}{q}} \\
& \cdot\left(\int_{t-\delta}^{t-\epsilon}\|f(s, v(s))\|^{p} d s\right)^{\frac{1}{p}} \\
& \leq \sum_{n=1}^{\infty}\left(\varphi_{\alpha}(\epsilon) \varphi_{\alpha}(\delta+n-1-\epsilon)+\varphi_{\alpha}(\delta+n-1)\right)\|f(\cdot, v(\cdot))\|_{S^{p}} \\
& +\left(\varphi_{\alpha}(\epsilon) \varphi_{\alpha}(0)+\varphi_{\alpha}(\epsilon)\right)(\delta-\epsilon)^{\frac{1}{q}}\|f(\cdot, v(\cdot))\|_{S^{p}} \\
& \leq\left(\varphi_{\alpha}(\epsilon) \varphi_{0}+\varphi_{0}\right)\|f(\cdot, v(\cdot))\|_{S^{p}} \\
& +\left(\varphi_{\alpha}(\epsilon) \varphi_{\alpha}(0)+\varphi_{\alpha}(\epsilon)\right)(\delta-\epsilon)^{\frac{1}{q}}\|f(\cdot, v(\cdot))\|_{S^{p}} .
\end{aligned}
$$

By using Lemma 3.1, we know

$$
S_{\alpha}(\epsilon) S_{\alpha}(t-s-\epsilon)-S_{\alpha}(t-s) \rightarrow 0, \quad \text { as } \epsilon \rightarrow 0 \text { for } s \in(-\infty, t-\delta]
$$

and

$$
\int_{-\infty}^{t-\delta}\left\|S_{\alpha}(\epsilon) S_{\alpha}(t-s-\epsilon)-S_{\alpha}(t-s)\right\|\|f(s, v(s))\| d s \leq\left(\varphi_{\alpha}(\epsilon) \varphi_{0}+\varphi_{0}\right)\|f(\cdot, v(\cdot))\|_{S^{p}} .
$$

Thus, by utilizing the arbitrariness of $\delta$ and the Lebesgue dominated convergence theorem, we obtain

$$
\lim _{\epsilon \rightarrow 0}\left\|S_{\alpha}(\epsilon) \int_{-\infty}^{t-\epsilon} S_{\alpha}(t-s-\epsilon) f(s, v(s)) d s-\int_{-\infty}^{t-\epsilon} S_{\alpha}(t-s) f(s, v(s)) d s\right\|=0 .
$$


Also,

$$
\begin{aligned}
\| S_{\alpha}(\epsilon) & \int_{-\infty}^{t-\epsilon} S_{\alpha}(t-s-\epsilon) f(s, v(s)) d s-\int_{-\infty}^{t} S_{\alpha}(t-s) f(s, v(s)) d s \| \\
\leq & \left\|S_{\alpha}(\epsilon) \int_{-\infty}^{t-\epsilon} S_{\alpha}(t-s-\epsilon) f(s, v(s)) d s-\int_{-\infty}^{t-\epsilon} S_{\alpha}(t-s) f(s, v(s)) d s\right\| \\
& +\left\|\int_{t-\epsilon}^{t} S_{\alpha}(t-s) f(s, v(s)) d s\right\| \\
\leq & \left\|S_{\alpha}(\epsilon) \int_{-\infty}^{t-\epsilon} S_{\alpha}(t-s-\epsilon) f(s, v(s)) d s-\int_{-\infty}^{t-\epsilon} S_{\alpha}(t-s) f(s, v(s)) d s\right\| \\
& +\int_{t-\epsilon}^{t} \varphi_{\alpha}(t-s)\|f(s, v(s))\| d s \\
\leq & \left\|S_{\alpha}(\epsilon) \int_{-\infty}^{t-\epsilon} S_{\alpha}(t-s-\epsilon) f(s, v(s)) d s-\int_{-\infty}^{t-\epsilon} S_{\alpha}(t-s) f(s, v(s)) d s\right\| \\
& +\left(\int_{t-\epsilon}^{t} \varphi_{\alpha}^{q}(t-s) d s\right)^{\frac{1}{q}} \cdot\left(\int_{t-\epsilon}^{t}\|f(s, v(s))\|^{p} d s\right)^{\frac{1}{p}} \\
\leq & \left\|S_{\alpha}(\epsilon) \int_{-\infty}^{t-\epsilon} S_{\alpha}(t-s-\epsilon) f(s, v(s)) d s-\int_{-\infty}^{t-\epsilon} S_{\alpha}(t-s) f(s, v(s)) d s\right\| \\
& +\varphi_{\alpha}(0) \cdot \epsilon^{\frac{1}{q}} \cdot\|f(\cdot, v(\cdot))\|_{S p}
\end{aligned}
$$

Thus,

$$
\lim _{\epsilon \rightarrow 0}\left\|S_{\alpha}(\epsilon) \int_{-\infty}^{t-\epsilon} S_{\alpha}(t-s-\epsilon) f(s, v(s)) d s-\int_{-\infty}^{t} S_{\alpha}(t-s) f(s, v(s)) d s\right\|=0
$$

which implies that $\left\{\int_{-\infty}^{t} S_{\alpha}(t-s) f(s, v(s)) d s: v \in B_{r}\right\}$ is relatively compact in $X$ by using the total boundedness. Hence, the set $\left\{(U v)(t): v \in B_{r}, r>0\right\}$ is relatively compact in X for every $t \in \mathbb{R}$. Thus, $U$ is completely continuous on $B_{r}$.

Now, the convex and closed hull of $U\left(B_{r}\right)$ is denoted by $\overline{c o} U\left(B_{r}\right)$. Since $U\left(B_{r}\right) \subset B_{r}$ and $B_{r}$ is convex and closed, $\overline{\mathrm{co}} U\left(B_{r}\right) \subset B_{r}$. Therefore, $U\left(\overline{\mathrm{co}} U\left(B_{r}\right)\right) \subset U\left(B_{r}\right) \subset \overline{\mathrm{co}} U\left(B_{r}\right)$. This means that $U: \overline{\mathrm{co}} U\left(B_{r}\right) \rightarrow \overline{\mathrm{co}} U\left(B_{r}\right)$ is a continuous mapping. It is easy to prove that, for each $t \in \mathbb{R},\left\{x(t): x \in \overline{\operatorname{co}} U\left(B_{r}\right)\right\}$ is relatively compact in $\mathbb{X}$, and $\overline{\operatorname{co}} U\left(B_{r}\right) \subset \mathrm{BC}(\mathbb{R}, \mathbb{X})$ is uniformly bound and equi-continuous since $\mathrm{UB}_{r}$ is. According to Arzela-Ascoli theorem, $\left\{x(t): x \in \overline{\mathrm{co}} U\left(B_{r}\right)\right\}_{t \in I}$ is relatively compact in $C(I, \mathbb{R})$, where $I$ is an arbitrary compact subset of $\mathbb{R}$. By $\left(\mathrm{A}_{5}\right),\left\{f\left(\cdot, v_{n}(\cdot)\right)\right\}$ is relatively compact in $\operatorname{PAA}^{p}(\mathbb{X}, \mu)$. Therefore there is a subsequence of $\left\{f\left(\cdot, v_{n}(\cdot)\right)\right\}$, recorded once more by $\left\{f\left(\cdot, v_{n}(\cdot)\right)\right\}$, which is convergent in $\operatorname{PAA}^{p}(\mathbb{X}, \mu)$, that is, for $\epsilon>0$, There is $N>0$ satisfying, for $m, n>N$,

$$
\left\|f\left(\cdot, v_{n}(\cdot)\right)-f\left(\cdot, v_{m}(\cdot)\right)\right\|_{S^{p}}<\frac{\epsilon}{\varphi_{0}} .
$$

For $m, n>N$, from (3.1), we have

$$
\left\|U v_{n}-U v_{m}\right\|=\sup _{t \in \mathbb{R}}\left\|U v_{n}(t)-U v_{m}(t)\right\| \leq \varphi_{0}\left\|f\left(\cdot, v_{n}(\cdot)\right)-f\left(\cdot, v_{m}(\cdot)\right)\right\|_{S^{p}}<\epsilon,
$$


which means that $\left\{U v_{n}\right\}$ is convergent in $\operatorname{PAA}(\mathbb{X}, \mu)$. Thus, $U: \overline{\operatorname{co}} U\left(B_{r}\right) \rightarrow \overline{\operatorname{co}} U\left(B_{r}\right)$ is a compact operator. By using Schauder's fixed point theorem, $U$ has a fixed point $v \in$ $\overline{\mathrm{co}} U\left(B_{r}\right)$. This is just a $\mu$-paa mild solution of Eq. (1.1) such that $\|v\|<r$.

\section{An example}

In order to conclude our article, we provide a briefness application to explain our abstract results.

Example 4.1 Let $A=-\varrho I, a(t)=\frac{\varrho}{4} \frac{t^{\alpha-1}}{\Gamma(\alpha)}, \varrho>0,0<\alpha<1$ and $f(t, v)=f_{1}(t, v)+f_{2}(t, v)$ where $f_{1}(t, v)=\sin \frac{1}{2+\cos t+\cos \pi t}, f_{2}(t, v)=\frac{1}{1+t^{2}} h(v)$ and $h(v)= \begin{cases}v \sin \frac{1}{v}, & v \neq 0, \\ 0, & v=0 .\end{cases}$

From Eq. (1.1), we obtain

$$
D^{\alpha} v(t)=-\varrho v(t)-\frac{\varrho^{2}}{4} \int_{-\infty}^{t} \frac{(t-s)^{\alpha-1}}{\Gamma(\alpha)} v(s) d s+f(t, v(t)), \quad t \in \mathbb{R} .
$$

From Example 4.17 of [18], we known that $A$ generates an $\alpha$-resolvent family $\left\{S_{\alpha}(t)\right\}_{t \geq 0}$ satisfying $S_{\alpha}(t)=(r * r)(t)$ and $S_{\alpha}(t) \in L^{1}\left(\mathbb{R}_{+}\right)$, where $r=t^{\frac{\alpha}{2}-1} E_{\alpha, \frac{\alpha}{2}}\left(-\frac{\varrho}{2} t^{\alpha}\right)$. Thus, it is easy to see that the $\alpha$-resolvent family $\left\{S_{\alpha}(t)\right\}_{t \geq 0}$ satisfy the assumption $\left(\mathrm{A}_{1}\right)$.

Note that the function $f \in \operatorname{PAA}^{p}(\mathbb{R} \times \mathbb{X}, \mu)$, with the measure $\mu$ whose Radon-Nikodym derivative $\rho$ is defined as

$$
\rho(t)= \begin{cases}e^{-t}, & t \in(0,+\infty), \\ 1, & t \in(-\infty, 0] .\end{cases}
$$

It is easy to prove that $\varepsilon\left(\mathbb{R} \times \mathbb{X}, L^{p}(0,1 ; \mathbb{X}), \mu\right)$ is translation invariant, thus (A) holds. Moreover, we can inspect that $f$ meets all requirements $\left(\mathrm{A}_{2}\right)-\left(\mathrm{A}_{5}\right)$. Then Eq. (4.1) has a mild solution in $\operatorname{PAA}(\mathbb{X}, \mu)$ by Theorem 3.1.

Obviously, $f$ does not fulfill any kind of "Lipschitz" type condition. Thus, the results in the literature $[20,21]$ with some "Lipschitz condition" are not inadequate.

Acknowledgements

This work is supported by a Grant of NNSF of China (No. 11471227, 11561077) and Scientific Research Fund of Sichuan

Provincial Education Department (No. 17ZB0370, 18ZB0512).

Competing interests

The authors declare that they have no competing interests.

Authors' contributions

All authors read and approved the final manuscript.

Author details

${ }^{1}$ Department of Mathematics, Sichuan University, Chengdu, P.R. China. ${ }^{2}$ Department of Mathematics, Sichuan University of Arts and Science, Dazhou, P.R. China.

\section{Publisher's Note}

Springer Nature remains neutral with regard to jurisdictional claims in published maps and institutional affiliations.

Received: 14 September 2017 Accepted: 7 February 2018 Published online: 15 February 2018

\section{References}

1. Baleanu, D., Jafari, H., Khan, H., Johnston, S.J.: Results for mild solution of fractional coupledhybrid boundary value problems. Open Math. 13,151-152 (2015)

2. Herzallah, M.A.E., El-Shahed, M., Baleanu, D.: Mild and strong solutions for a fractional nonlinear Neumann boundary value problem. J. Comput. Anal. Appl. 15, 341-352 (2013) 
3. Herzallah, M.A.E., Baleanu, D.: Existence of a periodic mild solution for a nonlinear fractional differential equation. Comput. Math. Appl. 64, 3059-3064 (2012)

4. Kilbas, A., Srivastava, H., Trujillo, J.: Theory and Applications of Fractional Differential Equations. North-Holland Mathematics Studies, vol. 204. Elsevier, Amsterdam (2006)

5. Podlubny, l.: Fractional Differential Equations. Academic Press, New York (1999)

6. Bochner, S.: A new approach to almost automorphicity. Proc. Natl. Acad. Sci. USA 48, 2039-2043 (1962)

7. Liang, J., Zhang, J., Xiao, T.J.: Composition of pseudo almost automorphic and asymptotically almost automorphic functions. J. Math. Anal. Appl. 340, 1493-1499 (2008)

8. Xiao, T.J., Liang, J., Zhang, J.: Psudo almost automorphic solutions to semilinear differential equations in Banach space. Semigroup Forum 76, 518-524 (2008)

9. Blot, J., Mophou, G.M., N'Guérékata, G.M., Pennequin, D.: Weighted pseudo almost automorphic functions and applications to abstract differential equations. Nonlinear Anal. 71, 903-909 (2009)

10. Xia, Z.N., Fan, M.: Weighted Stepanov-like pseudo almost automorphy and applications. Nonlinear Anal. 75 , 2378-2397 (2012)

11. Blot, J., Cieutat, P., Ezzinbi, K.: Measure theory and pseudo almost automorphic functions: new developments and aplications. Nonlinear Anal. 75, 2426-2447 (2012)

12. Chang, Y.K., Zhang, R., N'Guérékata, G.M.: Weighted pseudo almost automorphic mild solutions to semilinear fractional differential equations. Comput. Math. Appl. 64, 3160-3170 (2012)

13. Ding, H.S., Liang, J., Xiao, T.J.: Almost automorphic solutions to abstract fractional differential equations. Adv. Differ. Equ. 2010, Article ID 508374 (2010)

14. Chen, C.H., Li, H.X.: Composition of $S^{P}$-weighted pseudo almost automorphic functions and applications. Electron. J. Differ. Equ. 2014, 236 (2014)

15. He, B., Cao, J., Yang, B.: Weighted Stepanov-like pseudo-almost automorphic mild solutions for semilinear fractional differential equations. Adv. Differ. Equ. 2015, Article ID 74 (2015)

16. Mophou, G.M.: Weighted pseudo almost automorphic mild solutions to semilinear fractional differential equations. Appl. Math. Comput. 217, 7579-7587 (2011)

17. Xia, Z.N.: Weighted pseudo almost automorphic solutions of hyperbolic semilinear integro-differential equations. Nonlinear Anal. 95, 50-65 (2014)

18. Ponce, R.: Bounded mild solutions to fractional integro-differential equations in Banach spaces. Semigroup Forum 87 377-392 (2013)

19. Chang, Y.K., Zhang, M.J., Ponce, R.: Weighted pseudo almost automorphic solutions to a semilinear fractional differential equation with Stepanov-like weighted pseudo almost automorphic nonlinear term. Appl. Math. Comput. 257, 158-168 (2015)

20. Chang, Y.K., N'Guérékata, G.M., Zhang, R.: Stepanov-like weighted pseudo almost automorphic functions via measure theory. Bull. Malays. Math. Sci. Soc. 39, 1005-1041 (2016)

21. Akdad, A.N., Essebbar, B., Ezzinbi, K.: Composition theorems of Stepanov $\mu$-pseudo almost automorphic functions and applications to nonautonomous neutral evolution equations. Differ. Equ. Dyn. Syst. 25, 397-416 (2017)

22. Fan, Z.B.: Characterization of compactness for resolvents and its applications. Appl. Math. Comput. 232, 60-67 (2014)

\section{Submit your manuscript to a SpringerOpen ${ }^{\circ}$ journal and benefit from:}

- Convenient online submission

- Rigorous peer review

- Open access: articles freely available online

- High visibility within the field

Retaining the copyright to your article

Submit your next manuscript at $\gg$ springeropen.com 